ÉGYPTE

monde arabe

\section{Égypte/Monde arabe}

14 | 1993

Dits et écrits, mémoires et rites

\title{
La divination dans le rituel du zar égyptien
}

\section{Tiziana Battain}

\section{(2) OpenEdition}

\section{Journals}

Édition électronique

URL : https://journals.openedition.org/ema/572

DOI : 10.4000/ema.572

ISSN : 2090-7273

\section{Éditeur}

CEDEJ - Centre d'études et de documentation économiques juridiques et sociales

\section{Édition imprimée}

Date de publication : 30 juin 1993

Pagination : 103-112

ISSN : 1110-5097

\section{Référence électronique}

Tiziana Battain, « La divination dans le rituel du zar égyptien », Égypte/Monde arabe [En ligne], 14 | 1993, mis en ligne le 08 juillet 2008, consulté le 07 juillet 2022. URL : http://journals.openedition.org/ema/ 572 ; DOl : https://doi.org/10.4000/ema.572

Ce document a été généré automatiquement le 7 juillet 2022.

Tous droits réservés 


\title{
La divination dans le rituel du zar égyptien
}

\author{
Tiziana Battain
}

1 Rituel de possession, le zâr s'inscrit, dans l'ensemble des pratiques analogues, comme un rituel d'adorcisme. Des cérémonies sont organisées par les possédés tout au long de leur vie pour se concilier la bienveillance des esprits - appelés génériquement jinn ou asyad (seigneurs) - qui sont la source de leurs malheurs. Ces cérémonies variées se caractérisent essentiellement par l'offre à ces derniers, de nourriture et d'animaux sacrifiés. Des chants rituels (daqqa), interprétés par plusieurs groupes de musiciens utilisant différents instruments - notamment les tambourins, la darbouka, les castagnettes, la flûte et la lyre (tambura) - appellent les asyad à se manifester les uns après les autres durant la séance de zâr. L'adepte en transe enchaîne une danse rituelle censée représenter l'esprit qui le possède. L'officiant du zâr - homme ou femme appelé kudiyya. Joue le rôle intermédiaire et permet la communication entre le monde des humains et celui des esprits.

Dans le zâr égyptien, la divination ou kachf al-athâr (découverte des traces) représente une pratique fondamentale qui revêt la forme d'un rite complet, bien défini dans le temps et l'espace. Etape préliminaire à l'accomplissement des cérémonies, elle y occupe une place particulière en ce qu'elle sert à reconnaître les signes de la possession. A travers cette pratique, la kudiyya entre en contact avec les esprits et assure, notamment par le biais de l'oniromancie, une communication constante avec l'univers de ces derniers qui, de cette manière, font connaître aux possédés leurs volontés.

3 La divination permet non seulement d'identifier - donc de nommer - l'esprit ou les esprits responsables de la possession, mais encore de connaître les raisons de leur assaut et les remèdes, offrandes, sacrifices appropriés pour les apaiser. De fait, en deçà et au-delà de son ancrage rituel, la divination exprime le mode de relation existant entre les possédés et les esprits.

$4 \quad$ L'identification de l'esprit est une étape essentielle du rituel : on est possédé par un esprit singulier, doté d'un nom propre, d'un sexe, d'une religion, d'un pays d'origine. Il a dans la communauté des esprits un statut, une fonction, un rang bien définis. Il se 
caractérise par tels comportements et tels sentiments, tels goûts alimentaires, vestimentaires et ornementaux.

Il s'agit par conséquent, dans un premier temps, de cerner cet esprit dans toute sa complexité iconographique, psychologique et comportementale. Puis, dans un deuxième temps, il faut diagnostiquer la possession (c'est-à-dire établir l'étiologie et la pathologie des maladies et malheurs causés par l'esprit en question). Les esprits se tiennent dans des lieux domestiques ou publics bien particuliers et attaquent leurs victimes dans certaines parties du corps comme la tête, les yeux, le.coeur, le foie, à moins qu'ils ne provoquent la stérilité, les fausses couches ou la mort des nouveau-nés. Enfin, dans un troisième temps, on accomplira les rites destinés à les apaiser : à chaque esprit nommé correspondent des comportements rituels, des offrandes alimentaires et des sacrifices d'animaux spécifiques. Une fois les esprits identifiés, la kudiyya sera capable d'indiquer les moyens de satisfaire leurs désirs,

6 La nomination des esprits constitue un élément novateur par rapport à l'Islam orthodoxe. Dans les textes religieux, notamment le Coran, les esprits sont des entités indistinctes qui portent une appellation générale et collective telle que jinn ou arwah. L'apport d'une culture étrangère ${ }^{2} a$ induit un changement radical dans la perception des esprits et de leur relation au monde des humains. Le possédé se confond désormais avec les asyad personnifiés, ce qui crée entre les deux êtres une relation basée sur l'acceptation et l'identification et auquel correspond un type de rituel : c'est pourquoi on a défini plus haut le zâr comme un rituel d'adorcisme, qui tend à l'apaisement des esprits, et non comme un rituel d'exorcisme, qui implique le refus total de la possession. Notons enfin que dans le cas du zâr égyptien, le rituel prend la forme d'une alliance nuptiale. Aussi le déroulement des séquences et le langage utilisé lors de la cérémonie rappellent-ils de près les rites matrimoniaux.

Les modalités du Kachf al-athar

7 Sur le conseil de la famille ou des amis, le possédé, homme ou femme, se rend chez une kudiyya du quartier - ou chez une autre, plus célèbre, habitant ailleurs. Le plus souvent, un voisin ou un membre de la famille - mère ou frère dans le cas d'une jeune femme - l'y accompagne. Le possédé expose à la kudiyya ses troubles, malaises, visions ou cauchemars ; relèvent-ils de la possession ? C'est ce que celle-ci est censée déceler d'après les symptômes. Si ce n'est pas le cas, elle envoie le client chez un médecin. La plupart des malades s'adressent aussi bien à une kudiyya du zâr qu'à un médecin, un homme de religion musulman (faqih ou chaykh) ou un prêtre copte pour avoir leur avis et trouver le remède adéquat.

8 La kudiyya procède alors à l'opération du kachf al-athâr à l'aide de deux méthodes parallèles et complémentaires : le diagnostic musical, qui s'effectue pendant une séance dite hadra, et l'oniromancie.

Le diagnostic musical

9 Le « diagnostic musical », expression que nous avons empruntée à Rouget (1990: 93), n'est pas un terme complètement inconnu dans le vocabulaire adopté par certains officiants du zâr. Le souci actuel de modernité - sinon de scientificité - de leurs méthodes les amène souvent à utiliser des expressions empruntées à la médecine : ainsi la cérémonie de la hadra qui, durant la phase divinatoire, sert à identifier les esprits à l'aide des chants rituels, est-elle comparée à une séance de radiologie (le terme utilisé à ce propos est celui de achi'a, qui fait référence aux rayons $\mathrm{X}$ ). 
10 La hadra a lieu généralement une fois par semaine dans la maison dé la kudiyya. Son caractère de cérémonie publique, centrée sur la musique et la danse et où se déploie un vaste répertoire de daqqa, en fait le lieu idéal pour l'identification des esprits. Lors de l'émission des premières notes, les adeptes, poussés par l'esprit qui les possède, descendent dans le cercle formé par les participants. Le néophyte, sous la pression d'une atmosphère surchauffée et d'un rythme convulsif, succombera facilement à la transe et se jettera avec fougue dans la danse, subjugué par une musique ou des paroles évocatrices. Le rôle des musiciens consiste, en jouant daqqa après daqqa, à trouver la juste tonalité qui stimule la transe.

11 Chaque daqqa invoque un esprit spécifique et la transe qu'elle provoque est considérée comme le signe de l'emprise de cet esprit, lequel viendra s'ajouter à la pléthore d'esprits identifiés par le biais de l'oniromancie.

12 Les séances de hadra constituent également le meilleur moyen de se familiariser avec les esprits et d'assimiler le comportement rituel. Soutenu par les adeptes et sous l'oeil attentif de la kudiyya, le néophyte sera initié à une danse qui présente un caractère de complexité pouvant rendre difficiles les premières évolutions. Au début, la transe se présente sous la forme de comportements désordonnés accompagnés par des cris et des mouvements convulsifs et spasmodiques rappelant l'hystérie. Dans ces moments, la kudiyya empêche le possédé de faire des mouvements qui le représentent, en s'efforçant de contrôler le plus possible les manifestations de la transe spontanée.

13 A l'issue de trois séances, le néophyte est considéré comme prêt à affronter la cérémonie d'initiation.

L'oniromancie : al-layla al-tabitiyya

14 L'oniromancie est, dans le Monde arabe, un procédé oraculaire courant et ancien qui, considéré comme une révélation divine, se rattache à la prophétie (Coran : 12, 6, 12, 37). Mais la tradition distingue trois groupes d'intermédiaires de la révélation : « Les anges, inspirateurs et guides des vrais prophètes; ensuite, les démons, inspirateurs et séducteurs des devins et des faux prophètes; enfin, les jinn lesquels, conçus à l'instar des hommes, peuvent être de bons ou de mauvais informateurs. » (T. Fahd $1987: 68)^{3} \mathrm{La}$ divination cependant, en tant que faculté due à la possession, est clairement attestée dans le Coran ainsi que dans nombre de textes musulmans ${ }^{4}$. Al-Mas'udi, dans Murug. addahab (III, 34/sqq.) rapporte que des hommes prétendent être aidés par les jinn dans leurs facultés de prédiction car la pureté de leurs âmes leur permette communiquer avec les esprits.

E. G. Von Grunebaum (1967: 7-20) soutient que le rêve est perçu différemment chez les Occidentaux et chez les Arabes, au moins à l'époque moderne. Pour les premiers, le rêve reflète une réalité psychologique qui émerge du rêveur lui-même; pour les seconds, c'est une "réalité extérieure ». De plus, .Corbin (1967: 403-404) explique l'interprétation des songes visionnaires par les maitres soufi du fait d'une appréhension du monde inédite. Le rêve participe d'une réalité tierce appelée 'âlam almithâ, c'est-à-dire le mundus imaginalis, le monde de l'imaginaire ${ }^{5}$.

Si l'on admet l'existence d'une réalité autre, cachée et transcendante, le rêve en est la manifestation la plus adéquate. Il dévoile ainsi des symboles à interpréter et, par conséquent, il existe une classe de professionnels préposée à cet office. Ces personnes qui détiennent une position intermédiaire entre les deux mondes - tels les chefs de confréries, les faqih populaires - sont généralement considérées comme des majdûb ou 
majnûn, des «fous » frappés d'une bénédiction divine correspondant souvent à un état de déficience. Lecerf note que durant la période pré-islamique, les noms des devins étaient souvent associés à des infirmités physiques (telles l'hémiplégie et l'épilepsie), ce qui lui fait supposer qu'il y avait peut-être "un lien entre le fait d'être inapte aux fonctions normales de la société et le fait d'être apte aux fonctions supra-normales». Les kudiyya du zâr sont également des possédées frappées de toutes sortes d'infirmités physiques ou mentales. Néanmoins elles sont capables de gérer, même socialement, leur état et leur maladie.

Plus qu'une technique divinatoire appartenant au rituel du zâr, l'oniromancie résume en elle la divination. Ainsi, si le kachf al-athâr désigne globalement la pratique de la divination, l'expression définit plus précisément la technique de l'interprétation des rêves. Celle-ci existe en dehors du rituel du zâr et est couramment pratiquée en Égypte, aussi bien par les possédés que par les non-possédés. La kudiyya compte parmi ses clients non seulement les adeptes mais aussi leurs familles ou voisins, qui font appel à son aide dans des moments difficiles. Parfois, ils lui demandent de lire le athâr grâce à la méthode "al-layla al-tabitiyya ", que nous allons évoquer ci-après, ou bien ils lui racontent leurs songes à des fins d'interprétation. Le rêve, du fait qu'il n'est pas seulement induit par le rituel mais survient de manière spontanée et hors des séances cérémoniales, est donc considéré comme un mode de relation constant avec le monde des esprits.

La phase de la divination au moyen des songes est appelée al-layla at-tabitiyya ${ }^{6}$. Cette expression signifie " veiller la nuit en attendant la visite des esprits ». Ceux-ci peuvent se manifester dans les rêves aussi bien de l'officiant - au moyen de l'incubation - que du néophyte, à travers des signes que seule la kudiyya a la faculté d'interpréter.

La méthode de « l'incubation »

19 La kudiyya s'apprête à voir apparaître les esprits responsables de la possession de son client. Le contact sera établi durant le sommeil, par l'intermédiaire de l'esprit qui joue le rôle de "conseiller» ou de "tuteur ", ou est l'un des grands esprits du zâr. Cette opération requiert, de la part de la kudiyya, une retraite spirituelle dans la pièce de travail - souvent sa chambre à coucher- où elle garde tous ses instruments et objets rituels et qui est aussi considérée comme étant la demeure terrestre des asyad. La kudiyya, parfumée et vêtue de blanc, s'isole dans cette pièce où elle aura auparavant fait brûler de l'encens. Elle demande au client un objet personnel demeuré au contact de son corps (mouchoir, voile de tête, pantoufles ou bout de vêtement). Le possédé y aura enveloppé une amulette ou une subha (chapelet) ainsi que de l'argent.

La kudiyya encense sept fois ces objets en récitant la fatiha (première sourate du Coran) et en invoquant les noms des esprits, puis place les objets sous son oreiller. A côté de sa tête, elle pose aussi un morceau de sucre, du sel et du pain, en signe de bonne entente avec les esprits. Pendant trois nuits successives, la kudiyya entrera ainsi en contact avec les esprits et, le quatrième jour, le client viendra chercher les résultats de la divination. Les rêves du néophyte

21 Une autre méthode consiste à demander la participation active du possédé à cette opération. Ce dernier doit avaler, avant de se coucher, un morceau de sucre avec une gorgée d'eau de rose, pour favoriser le contact avec les esprits qui lui apparaîtront en rêve. La kudiyya interprétera alors les rêves de son client. Cette pratique est couramment utilisée avec les adeptes déjà initiés, lors d'une nouvelle maladie ou d'un état d'anxiété et surtout lors de la réitération d'une cérémonie. La kudiyya cherche ainsi 
à savoir si d'autres esprits sont venus s'ajouter aux précédents et quelles sont leurs exigences.

Les caractéristiques des différents esprits - vêtements, couleurs, bijoux - tels qu'elles apparaissent dans le rêve sont ensuite identifiées par la kudiyya. Cependant, la plupart des informateurs sont d'accord pour affirmer que ce sont les animaux domestiques et comestibles (poules, coqs, pigeons, brebis, moutons, chameaux...) qui caractérisent le mieux les esprits. Le lieu où apparaît le personnage ou l'animal du rêve a aussi son importance (cimetière, source, toilettes) car il peut indiquer le lieu où la possession s'est réalisée et, en conséquence, dévoiler les règles de comportement qui ont été violées par le possédé et ont provoqué l'irritation du jinn.

Rêver d'une fête, d'un mariage, de bougies allumées, d'une table dressée, d'instruments de musique, signifie que les esprits désirent une grande cérémonie accompagnée de daqqa. Rêver d'un chameau ou du port de vêtements blancs indique que l'adepte sera bientôt kudiyya à son tour.

Quelques sources écrites de l'oniromancie

A l'instar d'autres pays arabes, nombreux sont les manuels d'interprétation.des songes ayant une diffusion populaire en Égypte. Les deux livres les plus diffusés encore aujourd'hui sont ceux d'Ibn Chahin et d'Ibn Sirin (mort en 728 après J.-C.), dont l'utilisation était attestée au XIXe siècle par Lane qui vécut au Caire jusqu'en 1870 (Lane 1981 : 264). Ce dernier révèle que les Egyptiens de l'époque attribuaient au rêve une fonction prémonitoire. Il note, en effet, que « quand une personne dit à l'autre « j'ai fait un rêve", l'autre répond "c'est bien» ou "il plaît à Dieu»; dans le cas d'un cauchemar, il répond « 0 Dieu, la bénédiction sur Muhammad» et il crache trois fois par-dessus son épaule gauche pour éloigner le malheur » (ibid). Ibn Sirin était déjà renommé au XIVe siècle. Ibn Khaldoun écrivait dans sa Muqaddima: "Muhammad Ibn Sirin était le plus célèbre parmi les oniromanciens ; on nota par écrit des règles que les gens se sont transmises jusqu'à nos jours » (T.Fahd1967:342).

Dans son livre (1992:121-162), Ibn Sirin consacre de longs chapitres à l'interprétation des songes comportant des animaux. Il est intéressant de noter que dans ce texte, un rapport d'équivalence est établi entre les animaux et des traits de caractère humains, les animaux personnifiant les humains tout comme les esprits sont calqués sur leur image. De fait, l'oniromancie du zâr partage certaines similitudes avec l'interprétation des songes selon Ibn Sirin.

Que le système de représentation du zâr se soit élaboré ou non grâce à l'apport de ces textes, il reste que ce système renvoie à l'équation animal/caractère du personnage dans ce cas l'esprit - présente depuis des siècles dans la société égyptienne.

Cependant, l'imagerie du zâr est bien plus complexe que les interprétations qu'en donne Ibn Sirin et suppose l'association de nombreux éléments - animaux, couleurs, vêtements, bijoux, parfums, lieux, comportements, discours et autres - se référant à un esprit particulier ou à une modalité particulière de possession. Ces éléments s'entremêlent pour former le système de référence du rituel, tout en pouvant aussi servir à l'interprétation de songes.

Prééminence du kachf al-athâr sur les autres pratiques

Des pratiques divinatoires ou de voyance autres que le kachf al-athâr sont couramment utilisées en Égypte, comme la lecture des cartes à jouer (appelée en dialecte égyptien cutcina), du marc de café (fingan), des lignes de la main ('ilm af-kaff), la géomancie ('ilm al- 
raml), l'astrologie (nigma). Ces divers procédés nécessitent l'emploi de livres magiques ainsi que la connaissance du nom de la personne et de celui de sa mère.

Ces pratiques permettent surtout de connaître le destin d'un individu et la source de ses malheurs. Elles permettent également de savoir si ceux-ci dérivent du mauvais œil (hasad), de la sorcellerie (sihr), d'un sort ('amal ou sufla) ou de la possession (lamsa ou labsa). Bien que considérées comme mineures, elles sont aussi utilisées par les officiants du zâr?.

Cependant, dans les cas de possession, le kachf at-athâr est perçu comme le procédé de divination le plus efficace. Il est l'apanage des grandes kudiyya du zâr et détient, dans l'ordre hiérarchique des pouvoirs magiques dont jouit une kudiyya, la première position dans la mesure où, comme nous l'avons vu, il permet d'identifier et de nommer les esprits.

\section{BIBLIOGRAPHIE}

Battain T., « Musique de transe et mythe du fambura dans le rituel du zâr en Égypte ", Peuples Méditerranéens 56-57, juil-déc. 1991.

Corbin H., «Le songe visionnaire en spiritualité islamique », in R. Caillois et G. E. Von Grunebaum, Le rêve et les sociétés humaines. Gallimard, Paris, 1967, p. 380-406.

Fahd T., "Le rêve dans la société islamique du moyen âge ", in R. Caillois et G. E. von Grunebaum, op. cit., p. 335-365.

Fahd T., La divination arabe, Sindbad, Paris, 1987.

Grunebaum von E. G., «Introduction : La fonction culturelle du rêve dans l'islam classique », in RCaillois et G. E. Von GRunebaum, op. cit., p. 7-23.

Ibn Sirin, L'interprétation des rêves dans la tradition islamique, Alif, Lyon, 1992.

Lane E. W. Manners and customs of the modem Egyptians, Last-West Publication, Londres, 1981.

Lecerf J., «Le rêve dans la culture populaire arabe et islamique », in R. Caillois et G. E. Von Grunebaum, op. cit., p. 366-379.

Rouget G., La musique et la transe, Gallimard, Paris, 1990.

\section{NOTES}

1. Une exception a pourtant été relevée. T. Fahd (1987 : 73) cite Al-Jahiz (Hayawân I : 166) qui dit que les Arabes « prétendaient que tout grand poête ( $f a h l)$ avait un chaytan dont il n'était que le porte-parole. Ce chaytan pouvait avoir un nom propre (celui de Farazdaq s'appelait Amr). Il était censé être mâle ou femelle ».

2. Le zâr aurait été introduit en Égypte à la tin du siècle dernier par les esclaves d'origine africaine. Voir aussi T. Battain (1991:181-192). 
3. Selon al-Isfahani dans son Kitâb ai-Aghâni ( $3: 189)$, « la différence entre le prophète et le porte-parole (khatib) des jinn réside dans le fait que l'inspirateur du premier lui parte à l'oreille droite et lui impose des vêtements blancs, alors que celui du second lui parle à l'oreille gauche et exige des vêlements noirs. » Rapporté par T. Fahd (1987 : 77).

4. «Mahomet reconnaissait que le kâhin (devin) recevait son savoir d'un esprit par " possession » (majnûn) ou plutôt par relation personnelle avec un jinn, lequel épie ce qui se passe au ciel et le communique à son confident. Quand le Coran fut révélé à Mahomet, des anges auraient monté la garde au ciel afin d'empêcher les jinn d'espionner, en leur décochant une étoile filante. »Cf. Coran, 15, 15-18 ; 35, 6-9 ; 41, 12 ; 67, 5 (T. Fahd 1987: 66).

5. « II ne s'agit pas ici d'irréel. Le mundus imaginalis, c'est le monde des formes et images autonomes. C'est un monde parfaitement réel, qui préserve toute la richesse et la diversité du monde sensible, mais à l'état spirituel. L'organe qui appréhende ce monde, ce ne sont ni les sens physiques ni l'intellect pur, mais les « sens supra-sensibles ", essentiellement la conscience imaginative. Cette théorie de la connaissance Imaginative est fondamentale. Sur elle, en effet, repose la validité des perceptions supra-sensibles, celle des songes visionnaires... » (Corbin, 1967: 403-404).

6. De bâta = passer la nuit.

7. Au moment de l'investiture de la kudiyya, on interpelle l'esprit tutélaire qui aidera le nouvel officiant dans l'art de la divination, pour connaître la technique qu'il emploiera. Si cet esprit est Rokoch, la technique sera celle de la cutcina (les cartes) car celui-ci aime le jeu de cartes ; s'il s'agit d'un esprit chrétien, on utilisera le fingan (la lecture du marc de café ; mais cette technique est mal considérée car elle permet aussi d'agir pour le mal et non exclusivement pour le bien). Pour tes autres, l'officiant utilisera l'oniromancie.

INDEX

Mots-clés : islam, religion, soufisme, rituel, Coran, zar

\section{AUTEUR}

TIZIANA BATTAIN

Centre d'études yéménites, Sanaa 